\title{
BM] Global Health Increasing participation in a vector control campaign: a cluster randomised controlled evaluation of behavioural economic interventions in Peru
}

\author{
Alison M Buttenheim, ${ }^{1}$ Valerie A Paz-Soldán, ${ }^{2}$ Ricardo Castillo-Neyra, ${ }^{3}$ \\ Amparo M Toledo Vizcarra, ${ }^{4}$ Katty Borrini-Mayori, ${ }^{4}$ Molly McGuire, ${ }^{2}$ \\ Claudia Arevalo-Nieto, ${ }^{4}$ Kevin G Volpp, ${ }^{5}$ Dylan S Small, ${ }^{6}$ Jere R Behrman, ${ }^{7}$ \\ Cesar Naquira-Verlarde, ${ }^{8}$ Michael Z Levy ${ }^{3}$
}

To cite: Buttenheim AM, Paz-Soldán VA, Castillo-Neyra $R$, et al. Increasing participation in a vector control campaign: a cluster randomised controlled evaluation of behavioural economic interventions in Peru. BMJ Glob Health 2018;3:e000757. doi:10.1136/ bmjgh-2018-000757

Handling editor Seye Abimbola

- Additional material is published online only. To view please visit the journal online (http://dx.doi.org/10.1136/ bmjgh-2018-000757).

Received 2 February 2018 Revised 30 July 2018 Accepted 3 August 2018

Check for updates

(C) Author(s) (or their employer(s)) 2018. Re-use permitted under CC BY-NC. No commercial re-use. See rights and permissions. Published by BMJ.

For numbered affiliations see end of article.

Correspondence to Dr Alison M Buttenheim; abutt@nursing.upenn.edu

\section{ABSTRACT}

Objective To assess the efficacy of strategies informed by behavioural economics for increasing participation in a vector control campaign, compared with current practice. Design Pragmatic cluster randomised controlled trial. Setting Arequipa, Peru.

Participants 4922 households.

Interventions Households were randomised to one of four arms: advanced planning, leader recruitment, contingent group lotteries, or control.

Main outcome measures Participation (allowing the house to be sprayed with insecticide) during the vector control campaign.

Results In intent-to-treat analyses, none of the interventions increased participation compared with control (advanced planning adjusted OR (a0R) 1.07 (95\% $\mathrm{Cl} 0.87$ to 1.32); leader recruitment aOR $0.95(95 \% \mathrm{Cl}$ 0.78 to 1.15$)$; group lotteries aOR $1.12(95 \% \mathrm{Cl} 0.89$ to 1.39)). The interventions did not improve the efficiency of the campaign (additional minutes needed to spray house from generalised estimating equation regressions: advanced planning $1.08(95 \% \mathrm{Cl}-1.02$ to 3.17$)$; leader recruitment 3.91 (95\% Cl 1.85 to 5.97); group lotteries $3.51(95 \% \mathrm{Cl} 1.38$ to 5.64$))$ nor did it increase the odds that houses would be sprayed in an earlier versus a later stage of the campaign cycle (advanced planning aOR 0.94 (95\% Cl 0.76 to 1.25); leader recruitment aOR 0.68 (95\% $\mathrm{Cl} 0.55$ to 0.83$)$; group lotteries aOR $1.19(95 \% \mathrm{Cl} 0.96$ to 1.47)). A post hoc analysis suggested that advanced planning increased odds of participation compared with control among households who had declined to participate previously (aOR 2.50 (95\% Cl 1.41 to 4.43$)$ ).

Conclusions Achieving high levels of household participation is crucial for many disease prevention efforts. Our trial was not successful in improving participation compared with the existing campaign. The trial highlights persistent challenges to field experiments as well as lessons about the intervention design process, particularly understanding barriers to participation through a behavioural lens.

Trial registration number American Economic Association AEARCTR-0000620.

\section{Key questions}

What is already known?

- Low community participation in Chagas disease vector control campaigns can threaten campaign effectiveness.

- Observed and stated barriers to participation include high inconvenience costs, low perceived threat of the disease, no perceived consequences for refusal and lack of knowledge of neighbours' participation.

What are the new findings?

- We applied behavioural economics to the design of three interventions to increase participation in a Chagas disease vector control campaign by directly addressing behavioural roadblocks.

- In our study, none of the interventions achieved higher participation compared with the ongoing Ministry of Health campaign.

What do the new findings imply?

- Subsequent efforts may want to target specific insights from behavioural economics in order to design health behaviour change interventions in public and community health programmes.

\section{INTRODUCTION}

Chagas disease is a devastating disease and the most important and high-burden parasitic disease in the Americas. ${ }^{1-4}$ Between 6 and 8 million people, including 192000 Peruvians, are currently infected with Trypanosoma cruzi, the aetiological agent of the disease. ${ }^{56}$ Serological surveys of the Chagas disease burden in Arequipa, Peru, where our study is located, revealed a prevalence of $4.7 \%$ among schoolchildren in three districts of the city. ${ }^{7}$ The prevalence was similar in adults in a community-based serosurvey. ${ }^{8}$ T. cruzi is usually transmitted by contact with the faeces of triatomine insects, which, when infected, harbour the 
parasite in their guts. Acute infections have a 20\%-30\% probability of eventually progressing to cardiac or digestive forms of chronic Chagas disease, which are difficult to treat and often fatal.

Door-to-door household indoor residual spray (IRS) campaigns are a critical component of disease prevention and surveillance programme for Chagas disease, as they are for many other vectorborne diseases including malaria, dengue, Zika and chikungunya ${ }^{9}$ When coupled with simple and inexpensive household behaviours, IRS can drastically decrease or eliminate the presence of insect vectors from the home, protecting household members from illness. ${ }^{10-12}$ Since the early 1990 s, the Southern Cone initiative has targeted Triatoma infestans, the principal insect vector of T. cruzi in South America, for elimination. ${ }^{11}$ The Southern Cone Initiative is a multicountry effort to eliminate Chagas disease from southern South America. Plans and activities are implemented by Ministries of Health at the national or local level, and information sharing and impact evaluation are coordinated by the Pan American Health Organization. The key intervention of the Southern Cone Initiative is a door-to-door IRS campaign to eliminate the insect vector. Following this intervention, three countries (Chile, ${ }^{13}$ Brazil $^{14}$ and Uruguay) ${ }^{15}$ were declared free of $T$. cruzi transmission by T. infestans. Control of this vector in southern Peru ${ }^{716}$ and much of Bolivia ${ }^{17}$ has been more difficult, attributable at least in part to low rates of household participation in the door-to-door vector control campaign. ${ }^{18} 19$

For Chagas disease (as for other vectorborne diseases like Zika and dengue $)^{20}$ poor campaign participation undermines the effectiveness of evidence-based vector control efforts. To date, many vector control campaigns have relied on conventional health promotion and health behaviour change frameworks that assume that individuals form behavioural intentions based on a rational assessment of costs and benefits and consistently act in their own best interests. ${ }^{21}{ }^{22}$ Recently, health and development researchers have looked to the field of behavioural economics for new approaches to facilitate behaviour change. ${ }^{23}$ These approaches recognise the common biases and mental shortcuts that influence our decisions including present bias (the tendency to more heavily weight current vs future costs and benefits of an action), ${ }^{24}{ }^{25}$ attentional resource constraints ${ }^{26-28}$ and the power of social norms. ${ }^{29} 30$

Using these principles and others, researchers have designed strategies and interventions that leverage mental biases and shortcuts to achieve health-improving behaviour change. For example, commitment devices, which offer participants the opportunity to commit to a behaviour in advance, help counter present bias and dynamic inconsistency by voluntarily constraining future choices so that longer term interests are prioritised even in the setting of tempting alternatives. Commitment devices have contributed to successful weight loss and healthy eating programmes in the USA, ${ }^{31-33}$ as well as savings programmes in the Philippines ${ }^{34}$ and Kenya. ${ }^{35}$
Planning prompts, which encourage people to form a plan for a desired behaviour, such as 'When situation $\mathrm{X}$ arises, I will implement response Y,' counter present bias, address attention constraints and have been shown to improve voter turnout, ${ }^{36}$ influenza vaccination, ${ }^{37}$ oral hygiene behaviours ${ }^{38}$ and weight loss. ${ }^{39}$ Using peer opinion leaders (who influence the attitudes, beliefs and behaviours of their peers) to promote behaviour change leverages the importance of social norms, an approach that has been successful in HIV prevention and influenza vaccination. ${ }^{40} 41$

Behavioural insights have also generated considerable interest in how best to structure financial incentives to motivate health-related behaviour change. ${ }^{32} 33$ 42-46 Lotteries counter present bias by providing an immediate and tangible reward for future-oriented behaviour and have been shown to be effective for promoting medication adherence, ${ }^{43}$ weight loss, ${ }^{32} 33$ cholesterol reduction ${ }^{47}$ and immunisation ${ }^{48}$ in the USA, as well as HIV testing behaviour in Lesotho. ${ }^{49}$ Group-based lotteries, where a group is eligible for a prize only if all group members participate in the targeted behaviour, additionally take advantage of anticipated regret, social norms and peer pressure. $^{50}{ }^{51}$ While behavioural economic approaches have achieved compelling results for individual behaviours in controlled settings, fewer studies have been conducted of large-scale global health interventions such as vector control campaigns. ${ }^{23} 42$

\section{Objectives}

The purpose of this study was to evaluate three novel interventions that leverage behavioural economic insights to increase participation in a Chagas disease vector control campaign in Arequipa, Peru. We designed three interventions intended to increase participation in Arequipa's vector control campaign and evaluated their effectiveness compared with the current campaign in a pragmatic cluster randomised controlled trial of almost 5000 households.

\section{METHODS \\ Study setting}

The study was conducted in Arequipa, a city of nearly 1 million inhabitants in southern Peru. Vectorborne T. cruzi transmission cycles were well established in Arequipa in the early 2000s. Serosurveys conducted in 2004 in poorer communities revealed that $5.3 \%$ of children had become infected. ${ }^{16}{ }^{52}$ A door-to-door insecticide application programme launched in 2003 has been highly successful, as measured by the decrease in infestation rates across the city. ${ }^{18}$ The current Ministry of Health $(\mathrm{MOH})$ insecticide campaign has three phases. Following an initial survey phase, the 'attack' phase consists of vector control personnel visiting neighbourhoods targeted for control measures to apply insecticide (usually deltamethrin) in houses and peridomestic areas over two cycles (Cycle 1 and Cycle 2) at 6-month intervals. During the attack 
phase, houses are first visited by a health promoter from $\mathrm{MOH} 1$ day prior to treatment to inform them about the campaign and how to prepare their home (ie, large furniture moved away from walls, food and dishes put away). Promoters also confirm the homeowner's willingness to have the home treated and schedule an appointment for the following day. On the day of treatment, vector control personnel spend about 90 min spraying the home. Following treatment, household members are asked to ventilate the house for 2-3 hours. Cycle 2 of the attack phase is carried out in the same manner approximately 6 months later. For optimum campaign effects, all households are supposed to participate in both cycles. This ensures that any eggs that survive the first spray have hatched and are then killed during the second spray. Beginning approximately 6 months after the completion of Cycle 2 of the attack phase, treated neighbourhoods enter the third campaign phase, an extended period of active and passive surveillance for insect vector reinfestation. Our interventions were designed to increase participation in the attack phase of the campaign.

\section{Sample}

Eligible participants in the trial were residents of Arequipa's Alto Selva Alegre District living in neighbourhoods (localidades, or localities) selected for insecticide spraying by $\mathrm{MOH}$ based on insect vector infestation levels. This district was chosen as our study site based on campaign timing. Alto Selva Alegre is a heterogeneous district that includes urban and periurban neighbourhoods, with an estimated population of $82412^{53}$ in approximately 15000 households. Prior to spraying the district, $\mathrm{MOH}$ campaign staff evaluated the infestation level of each locality from preliminary survey data and decided to conduct blanket spraying (treating all homes) in 22 localities with $2 \%$ or more households infested and to do focal spraying (treating infested houses and those adjacent to infested houses) in the remaining localities with less than $2 \%$ of households infested. Our trial sample included all 5036 households in the selected localities, or about $38 \%$ of the households in Alto Selva Alegre. All households were targeted for Cycle 2 participation, regardless of participation in Cycle 1. The field trial was conducted from March to September 2015 during Cycle 2 of insecticide application in this district.

\section{Interventions}

Based on prior formative work ${ }^{19} 54$ and employing a behavioural design approach, we developed three interventions hypothesised to increase household participation compared with the existing campaign: advanced planning, block leader recruitment and contingent group lotteries. While all interventions addressed low perceived need for the Chagas disease campaign (a clear finding from our formative work) ${ }^{54}$ each was behaviourally designed to address specific participation barriers in different ways. Behavioural design is a systematic approach to defining a target behaviour, diagnosing behavioural bottlenecks that prevent uptake or engagement in the behaviour and using that diagnosis to design and test behaviourally informed interventions. ${ }^{55}$ Our behavioural design process, including detailed justifications for intervention designs, interventions designed that were proposed but rejected, and changes from original designs, are described fully elsewhere. ${ }^{56}$ We briefly summarise the interventions here.

The first intervention, Advanced Planning, was intended to address perceived difficulties in preparing the home for spraying and scheduling spraying at a convenient time for the household. ${ }^{1954}$ In our observations of prior campaigns, spray brigades often arrived at houses scheduled for treatment only to find that household members had not remembered the appointment and had not made the necessary preparations. This necessitated either waiting for preparations to be completed or moving on to the next scheduled house, compromising campaign efficiency. In the Advanced Planning intervention, households were given the opportunity to schedule the spray several days in advance during a convenient 2hour appointment window and to make a plan for their household based on this commitment. Planning prompts (in the form of a refrigerator magnet with space to write the plans) were also offered. Planning prompts were hypothesised to assist households in both making and following through with plans to move furniture and prepare the home; they also served as reminders of the appointment time.

The second intervention, Block Leader Recruitment, was designed to address mistrust of the campaign and its staff and a lack of awareness of neighbours' participation in the campaign, two themes that emerged clearly in our formative work. ${ }^{19} 54$ Formal community leaders (MOH-trained health promoters, block captains and elected community officials) and informal neighbourhood opinion leaders (corner store owners, day care coordinators) were recruited by study staff, attended a training session and were given promotional T-shirts, a clipboard, a phonecard with mobile phone credit and the same educational materials used by campaign staff. Recruiters were asked to promote the campaign to 10-12 households on their block through multiple visits, wear the campaign T-shirts regularly and share information about overall participation rates in the neighbourhood. This intervention leveraged prior research on the powerful role that descriptive and injunctive norms can play in shaping behaviour ${ }^{29}$ and on the specific influence of neighbourhood opinion leaders. ${ }^{4041}$

The third intervention, Contingent Group Lotteries, addressed perceived costs of participation (time, inconvenience) and encouraged neighbours to communicate with each other about participation. ${ }^{1954}$ Households were assigned to lottery groups of five to seven contiguous households (usually along the same block face). Lottery groups were randomly assigned a lottery number from 1 to 45 . To avoid any concerns about possible lottery rigging we used the national lottery drawing on a specified date 
a few weeks in the future. If the group's number was one of the seven drawn in the national lottery on that date (probability of winning $=16.7 \%$ ), each household in the group who had participated in the campaign received a voucher to a local hardware store (value $\$ 17$; expected value $\$ 2.84$ ( $\$ 17 \times 16.7 \%)$ conditional on individual household participation). If $100 \%$ of households in the group had participated, all households in the group received a hardware store voucher of twice the individual value (\$34; expected value $\$ 5.68(\$ 34 \times 16.7 \%)$ conditional on all households in the group participating). Financial incentives such as lotteries can be effective behaviour change strategies because they make future, intangible and probabilistic benefits of a behaviour more immediate and tangible. Lotteries further leverage base rate neglect, as most people will focus more on the numerator (the prize amount) and pay less attention to the denominator (the probability of winning). We employed a group lottery (vs an individual lottery design) to further leverage peer pressure.

For all three interventions, the research team carried out the activities that supplemented the existing campaign (eg, notifying households about the lottery, scheduling appointments in advance and recruiting and training neighbourhood peer leaders). $\mathrm{MOH}$ campaign personnel carried out the usual campaign across all interventions and control clusters. As our research team has done in districts treated earlier in the campaign, we also contributed staff to assist in promoting the campaign across all arms of the study.

\section{Study design}

We assessed the impact of the interventions on households' participation using a cluster-randomised controlled field trial. A clustered design was deemed most feasible for intervention delivery and most appropriate for minimising spillover and contamination effects. We divided the study area into 56 clusters of approximately $80-100$ households using a K-means algorithm. The clusters did not represent any geographical, public health, political or social unit. Clusters were matched into 14 groups of four similar clusters; within each group, clusters were then randomly assigned to the control arm or one of the three interventions, which resulted in 14 clusters assigned to each trial arm. To perform the matching we calculated the Mahalanobis distance among clusters based on two cluster-level variables (infestation and participation in the previous spraying campaign cycle 6 months prior) and assigned weights to these observed variables. ${ }^{5758}$ To estimate the weights, we used data sets from previous spraying campaigns in the city of Arequipa to build a logistic regression model and estimate the impact of infestation in the previous campaign and participation in the previous campaign on participation in a subsequent campaign. The exponentiated coefficients from this model were the weights used for the matching algorithm. Random assignment was carried out by team members who were not directly involved with intervention delivery or data collection. $\mathrm{MOH}$ personnel (promoters and vector control personnel) were not notified of assignment, but were not completely blinded to condition given their interactions with households in different arms. We obtained a waiver of informed consent for participation in the research study evaluating the three new interventions compared with the usual campaign. This waiver did not affect or change the MOH's usual process for obtaining consent to spray houses with insecticide, nor did it limit the household's right to refuse to respond to campaign surveys or refuse insecticide applications.

\section{Data collection}

We conducted the field trial during Cycle 2 of the attack phase of the campaign (March to September 2015). While our study was originally designed to improve participation in Cycle 1 of the attack phase, the campaign schedule (which we did not control) dictated that we run the trial during Cycle 2. As the $\mathrm{MOH}$ promoters and vector control personnel and our study staff proceeded through the study site, we systematically recorded campaign visits to each household, including Cycle 2 participation. For households refusing treatment, reasons for refusal were captured on a standardised eight-category instrument developed for a previous study. ${ }^{19}$ Data collection procedures were similar to previous studies in which we accompanied and supported $\mathrm{MOH}$ staff during the various stages. ${ }^{818195459}$ We merged Cycle 2 campaign and participation data with the household data collected during the preliminary survey and Cycle 1 participation data into a single database for analysis. To support fidelity measurement and to better interpret results, we also collected data on block leader recruitment and activities, lottery prize distribution and voucher redemption, and, in a subsample of households in the trial, measures of household awareness of and responses to the different interventions.

\section{Outcomes}

The primary outcome was a binary measure of household participation in the Cycle 2 insecticide application of the IRS campaign. Properties included on campaign maps were categorised in the field as treated, refused (household declined to allow the campaign to spray the house) or 'closed' (meaning no household member answered the door during campaign visits). We dichotomised household status into participated (treated) or not participated (refused or closed). Participation status of the household was assessed and recorded by campaign and study staff on household rosters at every visit, including visits conducted during 'campaign recovery efforts' in the month following the attack phase. For the purposes of this study, the outcome of interest was the last recorded participation status.

Two secondary outcomes related to campaign efficiency were also recorded: the number of minutes required to complete the insecticide application (calculated by subtracting the start time of the visit from the end time), and whether participating households were treated during the campaign's initial pass through the neighbourhood or 
during a later catch-up visit. This was assessed based on the date of treatment.

\section{Sample size and statistical methods}

An a priori sample size of 5600 households ( 1400 per arm) was calculated to provide $88.5 \%$ power to detect a difference in participation of 11 percentage points, assuming a baseline participation of $66 \%$ (as observed in a previously sprayed district), ${ }^{19}$ an alpha of 0.05 , and accounting for the clustered nature of the sample. The minimal detectable effect of 11 percentage points was determined to be of sufficient magnitude to be epidemiologically meaningful and operationally relevant to the ongoing $\mathrm{MOH}$ control campaign. ${ }^{60}$

Potential effects on participation by intervention arm were assessed first through two-way Pearson $\mathrm{X}^{2}$ test, with a Bonferroni-corrected critical value of $0.05 / 6=0.0083$ to account for six two-way comparisons of the four arms. We then estimated the marginal mean effect of each strategy using generalised estimating equations (GEE). We fit a logistic GEE model with an unstructured matrix to account for the correlation of houses within the same cluster. ${ }^{61} \mathrm{We}$ had 56 clusters in total, with 14 clusters for each arm and between 90 and 180 houses in each cluster. The GEE model included two prespecified household-level covariates: the household's Cycle 1 infestation (infested, not infested) and participation status (sprayed, refused or closed). We did not include in the GEE logistic model any cluster-level variable. Clusters were balanced as described above. This estimation strategy yields robust SEs. The inclusion of prior infestation and participation status of the household from Cycle 1 as covariates addresses any correlation in participation within household over time, which itself is likely to be associated with unobserved cluster fixed effects. We conducted a prespecified intent-to-treat (ITT) analysis with all eligible households, as well as a prespecified per protocol analysis, restricted to households who received a campaign visit.

\section{Ancillary and exploratory analyses}

During the trial in Alto Selva Alegre, the $\mathrm{MOH}$ also initiated the spray campaign in a neighbouring district, Cerro Colorado. This afforded us the opportunity to trial at least one intervention against the usual campaign during Cycle 1. In partnership with the $\mathrm{MOH}$, who were interested in potential scale-up of the most effective intervention, we selected the lottery intervention to trial in Cerro Colorado prior to the completion of the main trial in Alto Selva Alegre. The lottery arm was chosen given interest in its potential to increase participation, but also given concerns about its feasibility, acceptability and scale-up. A total of 1610 households in 17 clusters in Cerro Colorado were randomised to lottery or control for Cycle 1 of the attack phase using the same procedures as in the main trial, and similar campaign outcome data were collected from these households.

We also assessed two secondary outcomes related to overall campaign efficiency: conditional on being sprayed, we assessed whether the household was sprayed during the initial pass through the neighbourhood versus during 'catch up' or weekend campaign visits (as a proxy for ease of getting the house sprayed) and the number of minutes required to complete the insecticide application (as a proxy for how well the home had been prepared for spraying). We used linear regression fit with GEE to compare number of minutes required to spray the home and logistic regression fit with GEE to compare the timing of spray (initial wave or catch-up/weekend) across treatment arms. Secondary analyses were conducted for the ITT and per protocol samples; ITT results are described below. Given its programmatic relevance, we also conducted a post hoc exploratory analysis of participation by treatment arm only for those households in Alto Selva Alegre who had refused to participate in Cycle 1. The post hoc analysis used logistic regression fit with GEEs but did not include prior participation status as a covariate. Fidelity and process measures, reasons for refusal to participate and household responses to campaign interventions were tabulated using appropriate descriptive statistics. All analyses were carried out using Stata V.15.0.

\section{RESULTS}

We randomised 4974 eligible households from 22 localities in 14 clusters to treatment arms (figure 1). Excluding 48 houses lost to follow-up (for which we have no outcome measure) and four uninhabited houses, our ITT analysis included 4922 households. For per protocol analysis, we further excluded 556 households who did not receive a campaign visit. Baseline household characteristics were for the most part comparable across treatment arms in both ITT and per protocol samples (table 1). One notable exception was the higher proportion of vacant lots in the block leader recruitment arm $(4.1 \%$ vs $0.1 \%-0.2 \%$ in the other arms). Cycle 1 participation rates across treatment arms were $82.8 \%$ (control), $82.8 \%$ (advanced planning), $78.7 \%$ (block leader recruitment) and $81.8 \%$ (contingent group lottery).

\section{Participation by treatment arm}

Cycle 2 participation was high $(82.2 \%)$ in the study area (table 2), with little difference across study arms: $1052 / 1273(82.6 \%)$ in the control arm; $1057 / 1267$ $(83.4 \%)$ in advanced planning; 930/1180 (78.8\%) in block leader recruitment; and 1005/1202 (83.6\%) in contingent group lotteries (table 2). Participation in the unadjusted per protocol analysis was higher at $90.3 \%$ overall, with $91.1 \%$ in the control arm, $91.7 \%$ in advanced planning, $87.1 \%$ in block leader recruitment arm and $91.0 \%$ in contingent group lotteries. Pearson $\mathrm{X}^{2}$ tests suggest lower participation in the block leader recruitment arm compared with advanced planning and group lotteries in the ITT sample and lower participation compared with all other arms in the per protocol sample (table 2). In regression models estimating participation and controlling for covariates, no intervention arm was significantly different from 


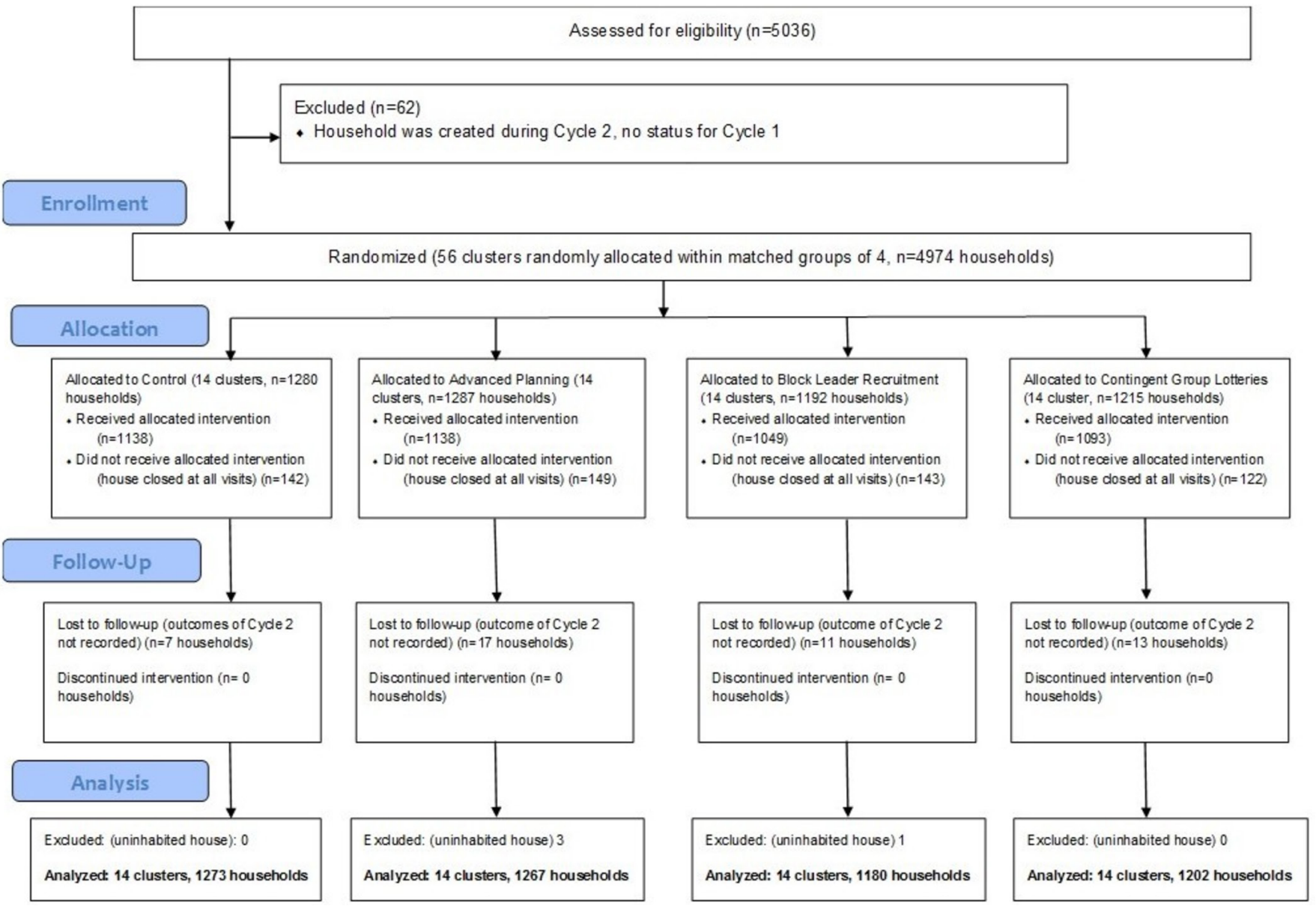

Figure 1 Consolidated Standards of Reporting Trials (CONSORT) flow diagram.

the control arm in either ITT or per protocol analyses (table 3). As expected, and consistent with our prior work, coefficients on control variables in our prespecified analyses show that participation was substantially higher (adjusted OR (aOR): 8.85, 95\% CI 4.43 to 17.70 ) in households in the ITT sample that were sprayed and were previously found to be positive for bugs, and also higher (aOR: 4.49, 95\% CI 3.33 to 6.06 ) in households that were sprayed but were found to be negative for bugs (uninfested) in Cycle 1. Houses that were uninhabited during Cycle 1 were also more likely to be treated (aOR: $2.19,95 \%$ CI 1.33 to 3.62 ), and lots recorded as public lots in the first treatment were less likely to be treated during Cycle 2 (aOR: $0.10,95 \%$ CI 0.02 to 0.45$)$. Households who had refused treatment in Cycle 1 were not significantly more or less likely to

Table 1 Baseline characteristics by treatment arm (n, \%)

\begin{tabular}{|c|c|c|c|c|c|c|c|}
\hline & $\begin{array}{l}\text { Control } \\
(n=1273)\end{array}$ & $\begin{array}{l}\text { Advanced } \\
\text { planning } \\
(n=1267)\end{array}$ & $\begin{array}{l}\text { Block leader } \\
\text { recruitment } \\
\text { ( } n=1180)\end{array}$ & $\begin{array}{l}\text { Contingent } \\
\text { group lottery } \\
(n=1202)\end{array}$ & $\begin{array}{l}\text { Total sample } \\
(n=4922)\end{array}$ & Pearson $\chi^{2}$ & $\begin{array}{l}P \\
\text { values }\end{array}$ \\
\hline \multicolumn{8}{|l|}{ Cycle 1 household status } \\
\hline Sprayed, infested & 49 (3.9\%) & 37 (2.9\%) & 39 (3.1\%) & $40(3.3 \%)$ & 165 (3.4\%) & 1.71 & 0.64 \\
\hline Sprayed, not infested & 1004 (78.9\%) & $1005(79.3 \%)$ & $892(75.6 \%)$ & 944 (78.5\%) & $3845(78.1 \%)$ & 6.01 & 0.11 \\
\hline Closed & $104(8.2 \%)$ & $94(7.4 \%)$ & 74 (6.3\%) & $80(6.7 \%)$ & $352(7.2 \%)$ & 3.94 & 0.27 \\
\hline Refused & $98(7.7 \%)$ & $106(8.4 \%)$ & $118(10.0 \%)$ & $123(10.2 \%)$ & 445 (9.0\%) & 6.89 & 0.08 \\
\hline Uninhabited & $12(0.9 \%)$ & $15(1.2 \%)$ & $8(0.7 \%)$ & $10(0.8 \%)$ & $45(0.9 \%)$ & 1.84 & 0.61 \\
\hline Vacant lot & $2(0.2 \%)$ & $1(0.1 \%)$ & $48(4.1 \%)$ & $2(0.2 \%)$ & $53(1.1 \%)$ & 130.4 & $<0.01$ \\
\hline Public lot & $4(0.3 \%)$ & $9(0.7 \%)$ & $1(0.1 \%)$ & $3(0.3 \%)$ & $17(0.4 \%)$ & 7.58 & 0.06 \\
\hline
\end{tabular}

Sprayed: house was treated with insecticide by campaign brigade. Infested: campaign staff found evidence of infestation by triatomine insects. Closed: no household member answered the door during campaign visits. Refused: household declined to allow the campaign to spray the house. 
Table 2 Pairwise comparison of proportion of households participating in insecticide treatment during Cycle 2 of attack phase Chagas disease vector control campaign, Arequipa, Peru, 2015, by treatment arm and analytic sample

\begin{tabular}{|ccc}
\hline Pairwise comparison & Pearson $\chi^{2}$ & P values \\
\hline Intent-to-treat & & \\
\hline Control (1052/1273, 82.6\%) versus advanced planning $(1057 / 1267,83.4 \%)$ & 0.278 & 0.598 \\
\hline Control (1052/1273, 82.6\%) versus block leader recruitment (930/1180, 78.8\%) & 5.778 & 0.016 \\
\hline Control (1052/1273, 82.6\%) versus contingent group lottery (1005/1202, 83.6\%) & 0.416 & 0.519 \\
\hline Advanced planning (1057/1267, 83.4\%) versus block leader recruitment (930/1180, 78.8\%) & 8.513 & 0.004 \\
\hline Advanced planning (1057/1267, 83.4\%) versus contingent group lottery (1005/1202, 83.6\%) & 0.015 & 0.901 \\
\hline Block leader recruitment (930/1180, 78.8\%) versus contingent group lottery (1005/1202, 83.6\%) & 8.989 & 0.003 \\
\hline Per protocol & & \\
\hline Control (1037/1138, 91.1\%) versus advanced planning (1044/1138, 91.7\%) & 0.275 & 0.600 \\
\hline Control (1037/1138, 91.1\%) versus block leader recruitment (914/1049, 87.1\%) & 9.046 & 0.003 \\
\hline Control (1037/1138, 91.1\%) versus contingent group lottery (995/1093, 91.0\%) & 0.006 & 0.940 \\
\hline Advanced planning (1044/1138, 91.7\%) versus block leader recruitment (914/1049, 87.1\%) & 12.371 & $<0.0001$ \\
\hline Advanced planning (1044/1138, 91.7\%) versus contingent group lottery (995/1093, 91.0\%) & 0.353 & 0.552 \\
\hline Block leader recruitment (914/1049, 87.1\%) versus contingent group lottery (995/1093, 91.0\%) & 8.412 & 0.004 \\
\hline
\end{tabular}

participate in Cycle 2 (aOR 1.48, 95\% CI 0.36 to 6.09). These results were directionally similar for the per protocol sample. Cluster-level participation rates by arm are shown in figure 2.

\section{Ancillary and secondary analyses}

The trial of the lottery arm for Cycle 1 in Cerro Colorado did not result in significant differences in the odds of participation compared with the control campaign ( $\mathrm{OR}=0.74,95 \%$ CI 0.45 to 1.21 , results not shown).

We hypothesised a priori that the advanced planning intervention may have improved the efficiency of the campaign by encouraging households to be better prepared for the sprayers' arrival, thereby reducing the amount of time the spray brigades had to spend in the home. In contrast to our hypothesis, no intervention group required fewer minutes to be sprayed than households in the control group (table 4). We also hypothesised that the interventions might encourage households to agree to participate sooner in the campaign (ie, not require additional promoter or spray brigade visits during weekend or 'catch-up' periods). In contrast, households in the block leader recruitment

\begin{tabular}{|c|c|c|}
\hline Outcome: household treated, Cycle 2 & Intent-to-treat aOR (95\% Cl) & Per protocol aOR(95\% Cl) \\
\hline \multicolumn{3}{|l|}{ Study arm (ref: control) } \\
\hline Advanced planning & 1.07 (0.87 to 1.32$)$ & 1.15 (0.78 to 1.69$)$ \\
\hline Block leader recruitment & 0.95 (0.78 to 1.15$)$ & 0.99 (0.72 to 1.37$)$ \\
\hline Contingent group lottery & 1.12 (0.89 to 1.39$)$ & 1.04 (0.76 to 1.42$)$ \\
\hline \multicolumn{3}{|l|}{ Cycle 1 household status (ref: closed) } \\
\hline Cycle 1: sprayed, positive & 8.85 (4.43 to 17.70$)$ & $12.86(4.13$ to 40.06$)$ \\
\hline Cycle 1: sprayed, negative & 4.49 (3.33 to 6.06$)$ & 3.87 (2.65 to 5.66$)$ \\
\hline Cycle 1: uninhabited & 2.19 (1.33 to 3.62$)$ & 8.10 (1.19 to 55.28$)$ \\
\hline Cycle 1: refused & $1.48(0.36$ to 6.09$)$ & 1.45 (0.31 to 6.83$)$ \\
\hline Cycle 1: public lot & $0.10(0.02$ to 0.45$)$ & 0.03 (0.01 to 0.12$)$ \\
\hline Cycle 1: vacant lot & 0.79 (0.57 to 1.10$)$ & 0.55 (0.35 to 0.87$)$ \\
\hline Constant & 1.54 (1.13 to 2.10$)$ & 3.84 (2.72 to 5.43$)$ \\
\hline n (households) & 4922 & 4418 \\
\hline
\end{tabular}

Adjusted ORs (aOR) from logistic regressions of the odds of households being treated (sprayed with insecticide) during Cycle 2 of the Chagas disease vector control campaign in Arequipa, Peru. Regression models are estimated using generalised estimating equations with robust SEs. Cls are adjusted for clustering of households within clusters. All covariates included in the model are shown in the table above. 


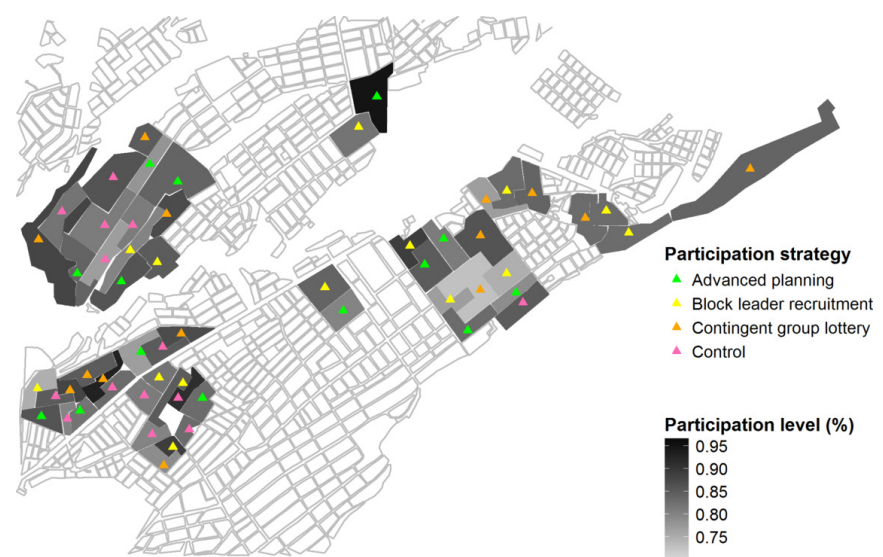

Figure 2 Map showing cluster-level participation rates in Cycle 2 of Chagas disease vector control campaign indoor residual spray campaign attack phase by intervention arm, Alto Selva Alegre District, Arequipa, Peru.

arm were less likely (aOR: $0.68,95 \%$ CI 0.55 to 0.83 ) to be sprayed during the initial spray period compared with control households (online supplementary table S1).

An exploratory analysis examined Cycle 2 participation among only those households who had refused to be sprayed in Cycle 1 of attack phase (vs being a 'closed' house that never answered the door at any promoter or sprayer visit). Among this subsample of

Table 4 Coefficients from ancillary analysis of efficiency of Chagas disease vector control campaign, Arequipa, Peru, 2015

\section{Minutes to spray house, among treated households B $(95 \% \mathrm{Cl})$}

\begin{tabular}{|c|c|}
\hline Study arm (ref: control) & $1.08(-1.02$ to 3.17$)$ \\
\hline Advanced planning & $3.91(1.85$ to 5.97$)$ \\
\hline Block leader recruitment & 3.51 (1.38 to 5.64) \\
\hline Contingent group lottery & $6.85(1.86$ to 11.84$)$ \\
\hline Cycle 1 household status (ref: closed) \\
\hline Cycle 1: sprayed, positive & $-1.49(-4.81$ to 1.82$)$ \\
\hline Cycle 1: sprayed, negative & $-13.47(-22.07$ to 4.86$)$ \\
\hline Cycle 1: uninhabited & $-11.79(-25.79$ to 2.22$)$ \\
\hline Cycle 1: refused & $-21.27(-39.41$ to 3.13$)$ \\
\hline Cycle 1: public lot & $-0.37(-4.78$ to 4.04$)$ \\
\hline Cycle 1: vacant lot & $52.55(49.13$ to 55.98$)$ \\
\hline Constant & 4010 \\
\hline $\mathrm{n}$ (households) & \\
\hline
\end{tabular}

Coefficients are from a linear regression of the duration of the household treatment (insecticide application) in minutes during Cycle 2 of the Chagas disease vector control campaign in Arequipa, Peru. Regression models are estimated using generalised estimating equations. Cls are adjusted for clustering of households. Unadjusted mean number of minutes to spray house in control arm=51.4 $\mathrm{min}$.
445 households, odds of participation were 2.5 times higher among the advanced planning treatment arm compared with control households (online supplementary table S1; aOR: $2.50,95 \%$ CI 1.41 to 4.43 ).

Analysis of fidelity measures indicated minimal problems with the delivery of the interventions. For example, $66 \%$ of households in the advanced planning arm who answered the door during the advanced planning visit scheduled a spray appointment in advance. In the block leader recruitment clusters where detailed data were collected on block leader activities, leaders were recruited in all clusters, ranging from 2 leaders for 73 households in one cluster to 8 leaders for 77 households in another cluster. Activity rosters for block leaders recorded visits to or conversations with most assigned households. Of 135 households who won lottery prizes, all but 20 redeemed the voucher at the hardware store.

We compared recorded reasons for refusing the insecticide spray in Cycle 2 across treatment arms (online supplementary table S2). The two most commonly stated reasons, consistent with our earlier research, ${ }^{19}$ were not having time to wait at home or miss work to accommodate the spraying, and allergies (meaning both allergic reactions to the insecticide and concerns that household members with other allergies would have negative reactions to the insecticide spray). Across the intervention arms, not having time to participate was mentioned less among the advanced planning $(17.5 \%)$ and lottery groups $(17.7 \%)$ compared with control $(27.0 \%)$ and block leader recruitment (30.5\%) arms. Allergy concerns were more common for control $(14.3 \%)$ and advanced planning $(14.0 \%)$ than for block leader recruitment $(10.2 \%)$ and contingent group lottery $(9.8 \%)$ arms.

We assessed potential spillover and contamination effects by surveying a subsample of households in all treatment arms several weeks after the Cycle 2 spraying. We asked whether they had been aware of other strategies used by the campaign to encourage participation in addition to the strategy they were exposed to. Awareness of no other strategies was high: $98.0 \%$ (control), 94.5\% (advanced planning), 88.8\% (block leader recruitment) and $97.4 \%$ (contingent group lotteries). The advanced planning and block leader recruitment arms were most aware of the lottery; the lottery arm was most aware of advanced planning. The other strategies reported by households in the control arm were not strategies actually used in the trial or by the campaign.

\section{DISCUSSION}

We tested the effect of three behaviourally informed interventions to improve participation in a Chagas disease vector control campaign in Arequipa, Peru, through a cluster randomised trial. The study was motivated by declines in participation observed in recently sprayed districts, ${ }^{19}$ and by the hypothesised potential of strategies 
informed by behavioural economics. Each of the trialled strategies-advanced planning, block leader recruitment and contingent group lotteries-was designed to address specific behavioural roadblocks to participation. In our trial of almost 5000 households experiencing the second of two cycles of door-to-door insecticide application, none of the strategies improved participation relative to the usual campaign. In a separate sample of households in another district experiencing the first cycle of the campaign, the lottery intervention also did not improve participation relative to the control. The strategies also did not appear to reduce the amount of time needed to spray each house, nor improve the pace with which households agreed to be sprayed. The only significant improvement in participation associated with the behaviourally informed strategies (in an exploratory analysis) was a 2.5 times higher odds of participation in the advanced planning arm compared with control, among only those households who had refused participation in the first cycle of spray. This is consistent with time and scheduling constraints being the most common stated reason for refusal to participate in the campaign.

Our results add to a slim literature on strategies to improve participation in community-based vector control efforts. Most prior work in this area comprises observational studies of community perceptions of vector control efforts including IRS campaigns. Prior research suggests that participation decisions are often driven by factors other than disease prevention, particularly civic duty. ${ }^{62} 63$ The most commonly cited barriers to participation included fear of theft during control activities, ${ }^{64}$ odours and allergic reactions to insecticide ${ }^{65}$ and logistical challenges. ${ }^{667}$ While community engagement and mobilisation activities were associated with higher rates of participation, ${ }^{68-70}$ these strategies have not been evaluated in a randomised trial.

We note two factors that may have contributed to our finding of no effect. First, as mentioned above, our interventions were originally designed to boost participation in Cycle 1 of insecticide spray, that is, in a "campaign naive' district. However, for practical reasons related to campaign timing and progression, we conducted the trial during Cycle 2, when households already had experience with campaign promotion and messaging and had already made a participation decision several months earlier. (We note that this was not true for our ancillary analysis of the lottery intervention vs control conducted during Cycle 1 in a neighbouring district, which also found no increase in participation.)

Second, our trial was also affected by an unexpected increase in participation achieved in both spray cycles and across all arms. ${ }^{19}$ This was partially endogenous to the trial: while we provided trained staff to assist in promoting the campaign as we have done in the past, this was the first time that our research team was focused specifically on improving participation (vs observing infestation patterns). We also suspect that Hawthorne effects may have come into play, as field personnel were closely monitored by our study staff during all campaign activities and across all arms of the study. An additional factor resulting in participation rates right around $80 \%$ across all arms emerged over the course of the trial in our study district. In an effort to keep the campaign moving forward, an official decided to require spray brigades to achieve $80 \%$ coverage before designating a locality as completed. This directive effectively (and perversely) placed a cap on participation rates-once $80 \%$ coverage in a locality was reached, brigades were free to leave the locality without expending marginal effort to get participation beyond $80 \%$. While our study arms were intentionally randomly distributed across localities, the net effect of this ad hoc quota on the locality level was to decrease the power of our study by homogenising coverage and compressing opportunities for any arm to exceed that quota. While the quota rule was not official policy nor formalised in the campaign protocol, it nevertheless was operationalised on the ground by campaign staff, a reminder of the pragmatic nature of this type of large-scale trial and the challenges of maintaining fidelity to protocol.

One important advantage of the pragmatic trial approach, however, is strong external validity. While trials incorporating financial incentives are often criticised for not being scalable or generalisable, we argue that the design of our lottery incentives and our collaboration with $\mathrm{MOH}$ through all phases of the trial ensured that, had they proved successful in boosting participation, the lottery incentives could have been implemented in the Arequipa campaign and elsewhere. The rapid deployment of mobile devices and applications ("apps") in public health campaign fieldwork also makes community-responsive scheduling and just-in-time dispatching of spray brigades more feasible, even in low-resource settings. Our block leader strategy was a specific example of the widespread use of community health workers ${ }^{71}$ and the purposive leveraging of social capital and social networks to support health behaviour change. ${ }^{72}$ We will further assess generalisability of our strategies in planned analyses of cost-effectiveness, household response to the strategies and potential crowding out of motivation to participate in public health campaigns associated with receipt of financial incentives. ${ }^{73-75}$

Our study is among the first to take an explicit behavioural design approach to the development and evaluation of strategies for global public health programmes including vector control. Our interventions were motivated by our own prior work suggesting that households were more likely to accept insecticide treatment when their neighbours had also participated; that households were inconvenienced by the logistics of the current campaign; and that households' knowledge of Chagas disease risk and transmission was low. ${ }^{194}$ Our failure to significantly increase participation may have emerged at several points in our process of behavioural design in this context: First, gaps in campaign participation may not be due primarily to behavioural roadblocks 
but rather to structural barriers. In other words, this may not be a problem that behavioural insights can help fix. Based on our prior experience with the campaign and our formative work, we think this is unlikely. Second, we may have misdiagnosed the behavioural roadblocks in our formative work or mismatched our intervention design (employing behavioural economic strategies) to the behavioural roadblocks to participation experienced by Arequipan households. This is certainly a possibility, and further refinement and systemisation of the behavioural diagnosis and design steps are needed to ensure a robust match of behaviourally informed interventions to target behaviours. Third, our interventions may have been appropriately matched to behavioural bottlenecks but simply not of sufficient 'dose' or salience to make a difference. We were not able to test in this pragmatic trial whether, for example, more visits from a block leader or a larger lottery prize could have made a difference. Lastly, fidelity to the interventions may have been imperfect, leading to confusion or misunderstanding among household members despite our efforts to describe the interventions in clear and simple terms; our fidelity measures, however, do not suggest this. Ultimately, our null results may reflect the practicalities of implementing feasible interventions in the context of an ongoing $\mathrm{MOH}$ campaign. Several interventions that were developed early in our process were abandoned due to low feasibility and acceptability. ${ }^{56}$

\section{Limitations}

Given the pragmatic nature of the trial, there were some deviations from normal campaign operations and from our trial plan. These included the additional campaign staff provided by the study team and the ad hoc quota on locality-level participation described above. In addition, the trial district experienced unexpected campaign stoppages several times, for weather delays, special promotional events and an active canine rabies outbreak. ${ }^{76}$ However, we have no reason to suspect that these factors differentially affected outcomes by study arm. Despite careful attention to balanced assignment of clusters to study arms, the block leader recruitment arm did end up with a disproportionate number of abandoned properties and vacant lots; relatedly, our field staff also observed anecdotally that several of the block leader recruitment clusters exhibited low social capital and social connectedness, which made recruitment of block leaders challenging. While our study design introduced the potential for spillover and contamination, few households reported awareness of campaign interventions (eg, lotteries or advanced planning) that they did not receive.

\section{CONCLUSIONS}

Achieving high levels of household participation is crucial for many community-based disease prevention efforts including door-to-door vector control campaigns. Our trial of behaviourally informed strategies to increase participation was not successful in doing this, with the possible exception of influencing households who had previously refused participation. The trial offers lessons for researchers and practitioners alike about how the intervention design process (particularly understanding barriers to participation from a behavioural lens) intersects with the challenges of pragmatic trials of existing health programmes. A great advantage of pragmatic trials is that they test effectiveness in practice, although this often results in smaller effect sizes than in smaller efficacy studies with greater control over design and delivery of interventions. Our results also prompt us to think about heterogeneous treatment effects in order to tailor and target interventions, particularly in large-scale programmes. We remain optimistic about the potential to apply behavioural insights to public health campaigns where household engagement is key to success and will continue to pursue this approach in ongoing research in vector and pest control, canine rabies vaccination and child anaemia.

\section{Author affiliations}

${ }^{1}$ Department of Family and Community Health, School of Nursing, University of Pennsylvania, Philadelphia, Pennsylvania, USA

${ }^{2}$ Global Community Health and Behavioral Sciences, Tulane University, New Orleans, Louisiana, USA

${ }^{3}$ Department of Biostatistics, Epidemiology and Informatics, University of Pennsylvania Perelman School of Medicine, Philadelphia, Pennsylvania, USA ${ }^{4}$ Zoonotic Disease Research Lab, OneHealth Unit, School of Public Health and Administration, Universidad Peruana Cayetano Heredia, Arequipa, Peru

${ }^{5}$ Medical Ethics and Health Policy, School of Medicine, University of Pennsylvania Perelman, Philadelphia, Pennsylvania, USA

${ }^{6}$ Department of Statistics, University of Pennsylvania Wharton School, Philadelphia, Pennsylvania, USA

${ }^{7}$ Department of Economics School of Arts and Sciences, University of Pennsylvania, Philadelphia, Pennsylvania, USA

${ }^{8}$ Universidad Peruana Cayetano Heredia, Arequipa, Peru

Acknowledgements The authors thank the members of the Chagas Disease Working Group in Arequipa who contributed and facilitated this work, including Fernando Malaga, Andy Catacora, Juan Cornejo del Carpio and Woodward Paja. We gratefully acknowledge the invaluable contributions of the Ministerio de Salud del Perú (MINSA), the Dirección General de Salud de las Personas (DGSP), the Estrategia Sanitaria Nacional de Prevención y Control de Enfermedades Metaxenicas y Otras Transmitidas por Vectores (ESNPCEMOTVS), the Dirección General de Salud Ambiental (DIGESA), the Gobierno Regional de Arequipa, and the Gerencia Regional de Salud de Arequipa (GRSA). We thank Sherri Kaplan for manuscript assistance.

Contributors AMB, MZL, VAPS, RCN, KGV, JRB, CNV and MZL contributed to conceptualising and designing the trial. All authors contributed to intervention design and trial implementation. AMB, VAPS, TCN, AMTV, KBM, MM, CA-N, and MZL contributed significantly to data acquisition. AMB, RCN, CAN, DSS and MZL conducted data cleaning and analysis. All authors provided comments towards drafts of the article and approved this version for submission.

Funding This study was funded by a grant from Eunice Kennedy Shriver National Institute of Child Health and Human Development (R01HD075869). Support for formative and pilot work was provided by grants from the University of Pennsylvania's University Research Foundation and Global Engagement Fund.

Disclaimer Funders of this study had no role in study design, data collection, data analysis, writing of the report or decision to submit the article for publication.

Competing interests None declared.

Patient consent Not required. 
Ethics approval Ethical approval was obtained from the Institutional Review Boards of the Universidad Peruana Cayetano Heredia, the University of Pennsylvania and Tulane University.

\section{Provenance and peer review Not commissioned; externally peer reviewed.}

Data sharing statement The full data set and statistical code are available from the corresponding author.

Open access This is an open access article distributed in accordance with the Creative Commons Attribution Non Commercial (CC BY-NC 4.0) license, which permits others to distribute, remix, adapt, build upon this work non-commercially, and license their derivative works on different terms, provided the original work is properly cited, appropriate credit is given, any changes made indicated, and the use is non-commercial. See: http://creativecommons.org/licenses/by-nc/4.0/

\section{REFERENCES}

1. World Health Organization. 2012.The world heath report 2004: changing history, 2004 http://www.who.int/whr/2004/en/ (accessed 30 Jan 2018).

2. Moncayo A. Chagas disease: current epidemiological trends after the interruption of vectorial and transfusional transmission in the Southern Cone countries. Mem Inst Oswaldo Cruz 2003:98:577-91.

3. Romanha AJ, Castro SL, Soeiro MN, et al. In vitro and in vivo experimental models for drug screening and development for Chagas disease. Mem Inst Oswaldo Cruz 2010;105:233-8.

4. World Health Organization. The world health report 2003, annex 2 , deaths by cause, sex and mortality stratum in WHO regions, estimates for 2002, 2003.

5. World Health Organization. Chagas disease (American trypanosomiasis), 2018

6. Bern C, Montgomery SP, Herwaldt BL, et al. Evaluation and treatment of chagas disease in the United States: a systematic review. JAMA 2007;298:2171-81.

7. Bowman NM, Kawai V, Levy MZ, et al. Chagas disease transmission in periurban communities of Arequipa, Peru. Clin Infect Dis 2008:46:1822-8.

8. Levy MZ, Small DS, Vilhena DA, et al. Retracing micro-epidemics of Chagas disease using epicenter regression. PLoS Comput Biol 2011;7:e1002146.

9. World Health Organization. 2006.Indoor residual spraying: use of indoor residual spraying for scaling up global malaria control and elimination http://www.who.int/malaria/publications/atoz/htm_mal_ 2006_1112/en/index.html (accessed 11 Feb 2013).

10. Ponce C. Current situation of Chagas disease in Central America. Mem Inst Oswaldo Cruz 2007;102(Suppl 1):41-4.

11. Dias JC. Southern Cone Initiative for the elimination of domestic populations of Triatoma infestans and the interruption of transfusional Chagas disease. Historical aspects, present situation, and perspectives. Mem I Oswaldo Cruz 2007:30:11-18.

12. Moncayo A, Ortiz Yanine MI. An update on Chagas disease (human American trypanosomiasis). Ann Trop Med Parasitol 2006;100:663-77.

13. Lorca M, García A, Contreras MC, et al. Evaluation of a Triatoma infestans elimination program by the decrease of Trypanosoma cruz infection frequency in children younger than 10 years, Chile, 19911998. Am J Trop Med Hyg 2001;65:861-4.

14. Silveira A, Vinhaes M. Elimination of vector-borne transmission of Chagas disease. Mem Inst Oswaldo Cruz 1999;94(Suppl 1):405-11.

15. Elimination of transmission of Chagas disease in southernmost Latin America. World Health Forum 1994:15:299-300.

16. Levy MZ, Bowman NM, Kawai V, et al. Periurban Trypanosoma cruzi-infected Triatoma infestans, Arequipa, Peru. Emerg Infect Dis 2006:12:1345-52.

17. World Health Organization. Maladie de Chagas en Amérique latine: le point épidémiologique basé sur les estimations de 2010. Wkly Epidemiol Rec 2015;90:33-44.

18. Barbu CM, Buttenheim AM, Pumahuanca ML, et al. Residual infestation and recolonization during urban Triatoma infestans Bug Control Campaign, Peru. Emerg Infect Dis 2014;20:2055-63.

19. Buttenheim AM, Paz-Soldan V, Barbu C, et al. Is participation contagious? Evidence from a household vector control campaign in urban Peru. J Epidemiol Community Health 2014;68:103-9.

20. Vazquez-Prokopec GM, Montgomery BL, Horne P, et al. Combining contact tracing with targeted indoor residual spraying significantly reduces dengue transmission. Sci Adv 2017;3:e1602024.

21. Ajzen I. The theory of planned behavior. Organ Behav Hum Decis Process 1991;50:179-211.
22. Prochaska JO, Velicer WF. The transtheoretical model of health behavior change. Am J Health Promot 1997:12:38-48.

23. The World Bank. 2015. World development report 2015: mind, society, and behavior http://www.worldbank.org/content/dam/ Worldbank/Publications/WDR/WDR\%202015/Chapter-8.pdf

24. O'Donoghue T, Rabin M. Doing it now or later. Am Econ Rev 1999;89:103-24.

25. Frederick S, Loewenstein G, O'donoghue T. Time discounting and time preference: a critical review. J Econ Lit 2002;40:351-401.

26. Milkman KL, Chugh D, Bazerman $\mathrm{MH}$. How can decision making be improved? Perspect Psychol Sci 2009;4:379-83.

27. Kahneman D. Attention and effort. Englewood Cliffs, NJ: PrenticeHall, 1973.

28. Wickens CD. Processing resources and attention. Damos DL, Multiple-task performance: Taylor and Francis, 1991:3-34.

29. Bicchieri C. The grammar of society: the nature and dynamics of social norms. New York: Cambridge University Press, 2006.

30. Reid AE, Cialdini RB, Aiken LS. Social norms and health behavior. Handbook of behavioral medicine: methods and applications. 3 , 2010:263

31. Milkman KL, Rogers T, Bazerman MH. I'll have the ice cream soon and the vegetables later: a study of online grocery purchases and order lead time. Mark Lett 2010;21:17-35.

32. Volpp KG, John LK, Troxel AB, et al. Financial incentivebased approaches for weight loss: a randomized trial. JAMA 2008; $300: 2631-7$

33. John LK, Loewenstein $G$, Troxel AB, et al. Financial incentives for extended weight loss: a randomized, controlled trial. J Gen Intern Med 2011;26:621-6.

34. Ashraf N, Karlan D, Yin W. Tying odysseus to the mast: evidence from a commitment savings product in the Philippines. Q J Econ 2006;121:635-72.

35. Dupas P, Robinson J. Why don't the poor save more? Evidence from health savings experiments. Am Econ Rev 2013;103:1138-71.

36. Nickerson DW, Rogers T. Do you have a voting plan?: implementation intentions, voter turnout, and organic plan making Psychol Sci 2010;21:194-9.

37. Milkman KL, Beshears J, Choi JJ, et al. Using implementation intentions prompts to enhance influenza vaccination rates. Proc Nat Acad Sci U S A 2011;108:10415-20.

38. Sniehotta FF, Araújo Soares V, Dombrowski SU. Randomized controlled trial of a one-minute intervention changing oral self-care behavior. J Dent Res 2007;86:641-5.

39. Luszczynska A, Sobczyk A, Abraham C. Planning to lose weight: randomized controlled trial of an implementation intention promp to enhance weight reduction among overweight and obese women. Health Psychol 2007;26:507-12.

40. Valente TW, Pumpuang P. Identifying opinion leaders to promote behavior change. Health Educ Behav 2007;34:881-96.

41. Kelly JA, St Lawrence JS, Diaz YE, et al. HIV risk behavior reduction following intervention with key opinion leaders of population: an experimental analysis. Am J Public Health 1991;81:168-71

42. de Walque $\mathrm{D}$. The use of financial incentives to prevent undesirable behaviors, 2018

43. Volpp KG, Loewenstein G, Troxel AB, et al. A test of financial incentives to improve warfarin adherence. BMC Health Serv Res 2008;8:e272

44. Volpp KG, Troxel AB, Pauly MV, et al. A randomized, controlled trial of financial incentives for smoking cessation. N Engl J Med 2009;360:699-709.

45. Volpp KG, Asch DA, Galvin R, et al. Redesigning employee health incentives--lessons from behavioral economics. N Engl J Med 2011;365:388-90.

46. Giuffrida A, Torgerson DJ. Should we pay the patient? Review of financial incentives to enhance patient compliance. BMJ 1997;315:703-7.

47. Asch DA, Troxel AB, Stewart WF, et al. Effect of financial incentives to physicians, patients, or both on lipid levels: a randomized clinical trial. JAMA 2015;314:1926-35.

48. Yokley JM, Glenwick DS. Increasing the immunization of preschool children; an evaluation of applied community interventions. J Appl Behav Anal 1984;17:313-25.

49. Bjorkman Nyqvist M, Corno L, de Walque D. Using lotteries to incentivize safer sexual behavior: evidence from a randomized controlled trial on HIV prevention, 2015.

50. Bacharach M, Gold N, Sugden R. Beyond individual choice: teams and frames in game theory. Princeton University Press: Princeton, 2006

51. JHW T, Zizzo DJ. Groups, cooperation and conflict in games. $J$ Socio Econ 2008;37:1-17. 
52. Levy MZ, Kawai V, Bowman NM, et al. Targeted screening strategies to detect Trypanosoma cruzi infection in children. PLoS Negl Trop Dis 2007;1:e103

53. Instituto Nacional de Estadistica e Informatica de Peru. 2018.Poblacion 2000 al 2015 http://proyectos.inei.gob.pe/web/ poblacion/ (accessed 30 Jan 2018).

54. Paz-Soldán VA, Bauer KM, Hunter GC, et al. To spray or not to spray? Understanding participation in an indoor residual spray campaign in Arequipa, Peru. Glob Public Health 2018;13:65-82.

55. Datta S, Mullainathan S. Behavioral design: a new approach to development policy. Rev Income Wealth 2014;60:7-35.

56. Buttenheim AM, Levy MZ, Castillo-Neyra R. 2018.A behavioral design approach to improving vector-control campaigns. osf.io/ k95wb (accessed 30 Jan 2018).

57. Greevy RA, Grijalva CG, Roumie CL, et al. Reweighted Mahalanobis distance matching for cluster-randomized trials with missing data. Pharmacoepidemiol Drug Saf 2012;21(Suppl 2):148-54.

58. Zhang K, Small DS. Comment: The essential role of pair matching in cluster-randomized experiments, with application to the Mexican Universal Health Insurance Evaluation. Stat Sci 2009;24:59-64.

59. Levy MZ, Malaga Chavez FS, Cornejo Del Carpio JG, et al. Rational spatio-temporal strategies for controlling a Chagas disease vector in urban environments. $J R$ Soc Interface 2010;7:1061-70.

60. Blitstein JL, Murray DM, Hannan PJ, et al. Increasing the degrees of freedom in future group randomized trials: the $\mathrm{df}^{\star}$ approach. Eval Rev 2005;29:268-86.

61. Diggle P, Heagerty P, Liang K-Y. Analysis of longitudinal data: Oxford University Press, 2002.

62. Montgomery CM, Munguambe K, Pool R. Group-based citizenship in the acceptance of indoor residual spraying (IRS) for malaria control in Mozambique. Soc Sci Med 2010;70:1648-55.

63. Munguambe K, Pool R, Montgomery C, et al. What drives community adherence to indoor residual spraying (IRS) against malaria in Manhiça district, rural Mozambique: a qualitative study. Malar J 2011;10:344.

64. Chiaravalloti Neto F, Baglini V, Cesarino MB, et al. O Programa de Controle do Dengue em São José do Rio Preto, São Paulo, Brasil: dificuldades para a atuação dos agentes e adesão da população. Cadernos de Saúde Pública 2007;23:1656-64.

65. Ingabire CM, Rulisa A, Van Kempen L, et al. Factors impeding the acceptability and use of malaria preventive measures: implications for malaria elimination in eastern Rwanda. Malar J 2015;14:136.

66. Sakeni M, Khorram A, Majdzadeh R, et al. Indoor residual spraying coverage and acceptability rates to control malaria and the householders' reasons of acceptance or rejection of spraying, in South-East of Iran. International Journal of Infection 2015;2.

67. Rodríguez AD, Penilla RP, Rodríguez MH, et al. Acceptability and perceived side effects of insecticide indoor residual spraying under different resistance management strategies. Salud Publica Mex 2006:48:317-24.

68. Chanda E, Masaninga F, Coleman M, et al. Integrated vector management: the Zambian experience. Malar J 2008;7:164.

69. Gürtler RE, Kitron U, Cecere MC, et al. Sustainable vector control and management of Chagas disease in the Gran Chaco, Argentina. Proc Natl Acad Sci U S A 2007;104:16194-9.

70. Hiwat H, Hardjopawiro LS, Takken W, et al. Novel strategies lead to pre-elimination of malaria in previously high-risk areas in Suriname, South America. Malar J 2012;11:10.

71. Perry HB, Zulliger R, Rogers MM. Community health workers in lowmiddle-, and high-income countries: an overview of their history, recent evolution, and current effectiveness. Annu Rev Public Health 2014;35:399-421.

72. Martire LM, Franks MM. The role of social networks in adult health: introduction to the special issue. Health Psychol 2014;33:501-4.

73. Deci EL, Koestner R, Ryan RM. A meta-analytic review of experiments examining the effects of extrinsic rewards on intrinsic motivation. Psychol Bull 1999;125:627-68.

74. Kreps DM. Intrinsic motivation and extrinsic incentives. Am Econ Rev 1997;87:359-64.

75. Wenemark M, Vernby A, Norberg AL. Can incentives undermine intrinsic motivation to participate in epidemiologic surveys? Eur J Epidemiol 2010;25:231-5.

76. Castillo-Neyra R, Brown J, Borrini K, et al. Barriers to dog rabies vaccination during an urban rabies outbreak: qualitative findings from Arequipa, Peru. PLoS Negl Trop Dis 2017;11:e0005460. 